\title{
Anomalous Origin of the Right Coronary Artery Associated with Congenital Bicuspid Aortic Valve
}

\author{
SHIGEAKI AOYAGI, SHIGEMITSU SUZUKI, KENICHI KOSUGA \\ AND KIROKU OHISHI
}

\author{
Department of Surgery, Kurume University School of Medicine, \\ Kurume, 830 Japan
}

Received for publication July 11, 1991

\begin{abstract}
Summary: Anomalous origin of the right coronary artery is an infrequent anomaly. A case with anomalous origin of the right coronary artery from the ascending aorta above the left sinus of Valsalva is described. This patient was also found to have a bicuspid aortic valve. The aortic valve was markedly calcified and stenotic. The aortic valve was replaced with a St. Jude Medical prosthesis.
\end{abstract}

Key words: bicuspid aortic valve - anomalous origin of the right coronary artery--aortic stenosis - aortic valve replacement - calcified aortic valve

\section{Introduction}

An anomalous origin of one or both coronary arteries has been reported to occur in $0.5 \%$ to $1.2 \%$ of all patients referred for cardiac catheterization (Engel et al. 1975; Razani, 1975; Chaintman et al. 1976). The most frequent anomaly is anomalous origin of the left circumflex from the right sinus of Valsalva or proximal right coronary artery (Alexander and Griffith, 1956). Anomalous origin of the right coronary artery from the left sinus of Valsalva is a less frequent coronary anomaly occurring in $0.19 \%$ of the patients (Chaintman et al. 1976). A patient was recently observed in whom the right coronary artery arose aberrantly from the ascending aorta above the left sinus of Valsalva. This patient was also found to have a bicuspid aortic valve.

\section{Casere port}

A 59 yeare-old Japanese woman was referred for cardiac cathererization for evaluations of palpitation and chest oppression. On admission, a harsh ejection systolic murmur of a grade VI/VI, which radiated to the neck, was heard at the second intercostal space along the right sternal border. Baseline laboratory data and chest $\mathrm{X}$-ray were within normal limits. The resting electrocardiogram showed evidence of left ventricular hypertrophy with deppression of ST segment on the lateral precordial leads. The two-dimensional and pulsed Doppler echocardiogram revealed that the aortic valve was calcified and bicuspid, and a $120 \mathrm{mmHg}$ peak systolic pressure gradient across the aortic valve. Cardiac catheterization showed that pulmonary artery wedge pressure was $12 \mathrm{mmHg}$, and aortic pressure was $155 / 85 \mathrm{mmHg}$ with mean of $115 \mathrm{mmHg}$,

Reprint requests to Dr. Aoyagi, Second Department of Surgery, Kurume University School of Medicine, 67 Asahi-machi, Kurume, 830 Japan 

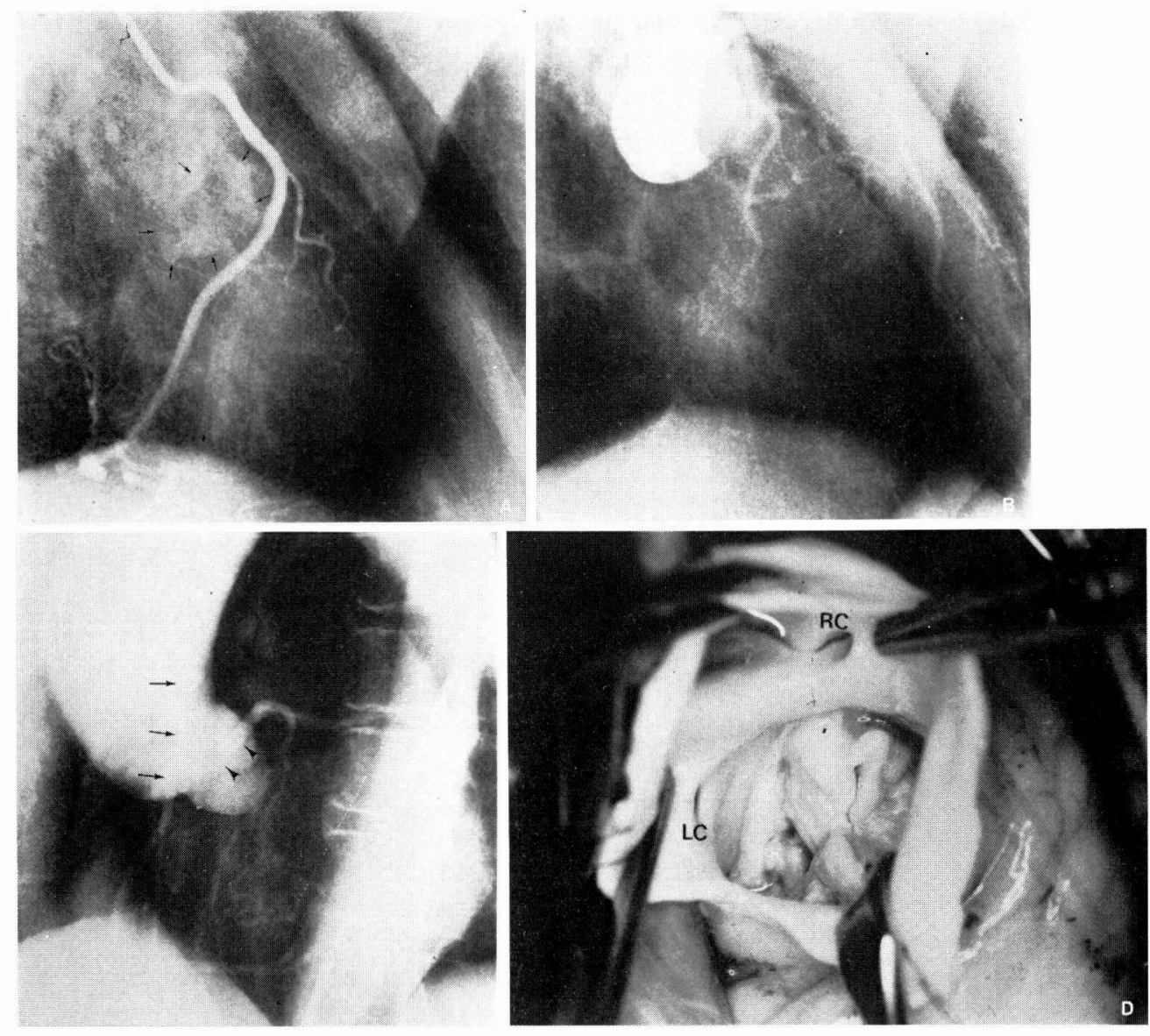

Fig. 1. A, Selective injection into the right coronary artery in the right anterior oblique projection demonstrating its origin from the aorta, high above the left sinus of Valsalva, and its anterior course between the aorta and the pulmonary artery. The arrows point to the calcified bicuspid aortic valve. B, A left coronary angiogram from the right sinus of Valsalva with a right anterior oblique projection. The left main coronary artery passed posterior to the aortic root and the pulmonary artery. The distal distribution of the left coronary artery was normal. C, Aortogram in the left anterior oblique projection showing the bicuspid aortic valve. The arrows point to the origin and course of the right coronary artery, and the arrow heads point to the origin and course of the left coronary artery. D, The aortic root showing the bicuspid aortic valve and the ostia of both coronary arteries. The origin of the right coronary artery was located high, about $2 \mathrm{~cm}$ above the rim of the left sinus of Valsalva, and the origin of the left coronary artery was in the aorta, immediately above the rim of the right sinus of Valsalva. RC; origin of the right coronary artery, LC; origin of the left coronary artery. 
however, the catheter was never inserted into the left ventricle. The aortogram showed the origin of the right coronary artery as above the left sinus of Valsalva, and there was no evidence of aortic insufficiency (Fig. 1C). On the selective coronary angiogram, the right coronary ostium was also located high above the sinus of Valsalva. This vessel coursed anteriorly between the aorta and the pulmonary artery to the right artio-ventricular groove (Fig. 1A). Although the left coronary ostium could not be cannulated, the coronary angiogram from the right sinus of Valsalva revealed that the origin of the left coronary was located immediately above the sinus of Valsalva. The left main coronary artery passed posterior to the aortic root and the pulmonary artery. The distal distribution of the left coronary artery was normal (Fig. 1B). At the operation, it was confirmed that the bicuspid aortic valve was markedly calcified, and the origin of the right coronary artery was located high about $2 \mathrm{~cm}$ above the rim of the left sinus of Valsalva, and the origin of the left coronary artery was in the aorta immediately above the rim of the right sinus of Valsalva (Fig. 1D). The calcified aortic valve was replaced with a St. Jude Medical prosthesis. The patient's postoperative course was uneventful.

\section{Discussion}

Origin of the right coronary artery from the left sinus of Valsalva is rare. In the series by Engel et al. (1975) only three of 51 patients with anomalous origin of the coronary arteries had an anomalous right coronary artery; and all arose from the left sinus anterior to the left coronary artery. In a group of 31 patients with anomalous coronary artery origins, Chaintman et al. (1976) found 7 with an aberrant origin of the right cor- onary artery.

Only two case reports of the right coronary artery arising from the aorta above the left sinus of Valsalva have previously been published (King et al. 1982; Palomo et al. 1985). All three patients, including this patient, were associated with bicuspid aortic valves. There is an increased variation of left coronary anatomy associated with a bicuspid aortic valve. This observation suggests the possibility that anomalies of the right coronary artery may also be associated with a bicuspid aortic valve. In these three cases, the right coronary artery coursed anteriorly between the aorta and the pulmonary artery. Anomalous origin of the right coronary artery from the left sinus of Valsalva have been considered benign as compared to anomalous origin of the left coronary artery (Chaintman et al. 1976). However, recently, manifestations of myocardial ischemia attributed to compression of the right coronary artery passing between the two great arteries, have been described in patients with this coronary anomaly in the absence of additional atherosclerotic or other disease processes (Brandt et al. 1983).

For the surgical approach to a patient with this anomaly, injection of cardioplegic solution into both coronary ostia, and implantation of a prosthetic valve in the native aortic annulus are easily performed; however, it is important to avoid severing an aberrant right coronary artery, especially when a patient requires enlargement of the aortic annulus for the treatment of the aortic valve disease.

\section{References}

Alexander, R.W. and Griffith, G.C. (1956). Anomalies of the coronary arteries and their clinical significance. Circulation 14, 800-805, Brandt, B.III., Martins, J.B. and Marcus, M.L. (1983). Anomalous origin of the right coronary artery from the left sinus of Valsalva. 
N. Engl. J. Med. 309, 596-598.

Chaintman, B.R., Lesperance, J., Saltiel, J. and Bourassa, M.G. (1976). Clinical, angiographic, and hemodynamic findings in patients with anomalous origin of the coronary arteries. Circulation 53, 122-131.

Engel, H. J., Torres, C. and Page, H.L. (1975). Major variations in anatomical origin of the coronary arteries. Cathet. Cardiovasc. Diag. 1, 157-169.

King, B.D., Ambrose, J. A., Stein, J.H., Ro, J.H. and Herman, M.V. (1982). Anomalous origin of the right coronary artery from the ascending aorta above the left coronary sinus. Cathet. Cardiovasc. Diag. 8, 277-280.

Palomo, A.R., Schranger, B.R. and Chahine, R. A. (1985). Anomalous origin of the right coronary artery from the ascending aorta high above the left posterior sinus of Valsalva of a bicuspid aortic valve. Am. Heart J. 109, 902-904.

RAZANI, M. (1975). Unusual forms of coronary artery disease. Cardiovasc. Clin. 1, 25-46. 